\title{
Inadvertent and minimal gluten intake has a negligible role in the onset of symptoms in patients with coeliac disease on a gluten-free diet
}

\author{
Annalisa Schiepatti ${ }^{1} \dagger$, Valentina Bellani ${ }^{1}$, Margherita Perlato ${ }^{1}$, Stiliano Maimaris ${ }^{1}$, Catherine Klersy ${ }^{2}$ and \\ Federico Biagi $^{{ }^{*}}$ \\ ${ }^{1}$ Coeliac Centre/First Department of Internal Medicine, University of Pavia, 27100 Pavia, Italy \\ ${ }^{2}$ Biometry and Clinical Epidemiology, Fondazione IRCCS Policlinico San Matteo, 27100 Pavia, Italy
}

(Submitted 28 August 2018 - Final revision received 29 October 2018 - Accepted 21 November 2018 - First published online 17 January 2019)

\begin{abstract}
Although the quantity of gluten that a well-instructed coeliac patient can involuntarily ingest is $<10 \mathrm{mg}$ of gluten/d which cannot induce significant villous damage, coeliac patients often attribute the origin of symptoms to the involuntary ingestion of trace quantities of gluten, either certain or hypothetical. Our aim was to evaluate whether the occurrence of symptoms in coeliac patients who histologically responded to a strict gluten-free diet was related to the involuntary consumption of minimal quantities of gluten. A case-control study to assess the association between gluten exposure and the occurrence of symptoms was designed. Between January 2017 and May 2018, coeliac patients attending our outpatient clinic were interviewed to detect the presence of symptoms. Based on a specifically designed form, patients were also divided into different risk groups of gluten exposure. A total of ninety-five coeliac patients on a strict gluten-free diet and with known histological recovery were enroled. Of them, fifty-two of them reported symptoms and they were enroled as cases; the remaining forty-three patients denied symptoms and were enroled as controls. Although this was not statistically significant, gluten exposure was more frequent in controls (Fisher's exact test, $P=0 \cdot 07$ ). Our results show no relationship between exposure to minimal quantities of gluten and onset of symptoms in coeliac patients. Symptoms are more frequent in patients who have no risk of gluten exposure. It is possible that the presence of these symptoms leads the patients to avoid situations that may place them at risk of gluten exposure.
\end{abstract}

Key words: Coeliac disease: Gluten: Gluten-free diet adherence

Coeliac disease (CD) is a chronic enteropathy due, in genetically susceptible individuals, to gluten, the complex of waterinsoluble proteins from wheat, rye and barley. In most patients with $\mathrm{CD}$, the enteropathy will reverse on a gluten-free diet $(G F D)^{(1)}$. However, a GFD is expensive and, to be effective, GFD must be followed very strictly and maintained indefinitely. Moreover, adherence rates to a GFD have been reported to vary between 40 and $90 \%$, and it is well known that dietary transgressions are the commonest cause for non-responsive and complicated $\mathrm{CD}^{(2,3)}$. Therefore, a careful evaluation of GFD adherence is a mandatory step in delivering follow-up care to coeliac patients. Although numerous methods have been proposed to assess GFD compliance, the best tool for disease monitoring is still a matter of debate ${ }^{(4,5)}$

A duodenal biopsy is certainly the most accurate tool to verify a good histological response and thus to understand whether the patient is on a strict GFD ${ }^{(4-6)}$. However, a duodenal biopsy requires an upper gastrointestinal endoscopy, an expensive and theoretically hazardous procedure. Coeliac-specific antibodies, a great tool for the diagnosis of $\mathrm{CD}^{(7,8)}$, are less useful for the follow-up of this condition ${ }^{(9)}$. Nevertheless, disappearance of serum coeliac-specific antibodies is one of the most commonly used tools to follow up coeliac patients ${ }^{(4,5)}$. Nowadays, the 'gold standard' for the follow-up of coeliac patients and for assessing GFD compliance is data collection by the patient, which is then discussed during a dietary interview. At the end, a doctor or an expert dietitian expresses a subjective evaluation on the patient's compliance with the $\mathrm{GFD}^{(1,4,5)}$.

A few years ago, others and we developed scoring systems aiming to evaluate the degree of GFD compliance ${ }^{(10-12)}$. The score we developed here in Pavia does not take into account the quantity of gluten consumed by the patient but focuses on the defensive strategies that the patient undertakes to avoid gluten ingestion. This score can be used, however, only in patients that were previously adequately instructed on what a GFD means and that are telling the $\operatorname{truth}^{(10)}$. In the same paper, we also showed that the quantity of gluten that a sensible, dietconscious and well-instructed patient can involuntarily ingest is

\footnotetext{
Abbreviations: CD, coeliac disease; GFD, gluten-free diet
}

* Corresponding author: F. Biagi, fax +39 0382 502618, email f.biagi@smatteo.pv.it

$\dagger \mathrm{PhD}$ course in Experimental Medicine, University of Pavia. 
really very small. It is much smaller than not only the quantity of $50 \mathrm{mg} / \mathrm{d}$ shown to induce villous damage but also than the quantity of $10 \mathrm{mg}$ of gluten/d which cannot induce significant intestinal damage ${ }^{(13,14)}$. On the basis of these results, we concluded that the involuntary ingestion of minimal quantities of gluten was not harmful for coeliac patients ${ }^{(10,11)}$.

In everyday clinical practice, coeliac patients often complain of symptoms and they attribute the origin of these symptoms to the involuntary ingestion of trace quantities of gluten. Sometimes the ingestion of gluten is certain and was due to an involuntary mistake. Other times the ingestion of gluten is, however, not certain and a hypothetical, involuntary ingestion is assumed by the patient to justify symptoms arising after consumption of food that theoretically could have been contaminated by gluten ${ }^{(15)}$. Finally, clinical trials evaluating adjunctive therapies to GFD-enroled patients on the basis of persisting symptoms, assuming that these symptoms were due to inadvertent ingestion of a minimal quantity of gluten are reviewed in Kurada et al. ${ }^{(16)}$

We are not certain that inadvertent gluten intake can induce symptoms in coeliac patients. Our clinical experience seems to suggest exactly the opposite. We described a patient in whom a clinical and serological recovery occurred, despite the regular consumption of very less quantity of gluten; nevertheless, there was no histological response ${ }^{(17)}$. Moreover, a double-blinded study performed on coeliac patients receiving either a minimal quantity of gluten or placebo showed that although villous damage was detectable in coeliac patients on gluten, no differences were evident as far as symptoms were concerned ${ }^{(13)}$.

The aim of our study is to evaluate whether the occurrence of symptoms in coeliac patients with evidence of histological recovery after at least 12 months on a strict GFD is related to the involuntary consumption of small quantities of gluten, either certain or hypothetical.

\section{Methods}

\section{Study design}

We designed a case-control study (unmatched) to assess the association of gluten exposure and the occurrence of symptoms.

\section{Data collection}

The study was performed on all coeliac patients directly diagnosed in our centre, on a GFD for at least 1 year and attending our outpatient clinic between January 2017 and May 2018.

Original diagnosis of CD was based on a certain degree of villous atrophy shown in a well-oriented duodenal biopsy and positive IgA endomysial antibodies. For patients with total IgA deficiency, class IgG antibodies were considered ${ }^{(1)}$. More precisely, we routinely take four duodenal biopsies from the second part of the duodenum during a gastroscopy performed under mild sedation (midazolam $0.05 \mathrm{mg} / \mathrm{kg}$ intravenous). Biopsies are always oriented on cellulose nitrate paper, formalin fixed and then paraffin embedded and cut perpendicularly with respect to the luminal surface. Endomysial antibodies are detected on monkey oesophagus/jejunum sections using an indirect immunofluorescence kit (INOVA Diagnostic). In our study we do not routinely test for tissue transglutaminase antibodies. In our experience, both sensitivity and specificity of endomysial and tissue transglutaminase antibodies are very similar and satisfactory, as previously described ${ }^{(18)}$. Patients were then instructed by expert personnel on how to follow a strict GFD. Clinical, histological and serological responses were re-evaluated in all of them $12-15$ months after the initial diagnosis $^{(19)}$. We underline that re-evaluation of most patients took place a few years before our current study.

To exclude the possibility that symptoms could be due to gross gluten contamination causing an unsatisfactory histological and clinical response, we did not take into account patients that were not on a strict GFD (Pavia score $<3^{(10)}$ ), patients with unsatisfactory histological response at the time of re-evaluation and patients with a complicated form of CD. Patients in whom symptoms were due to other conditions unrelated to CD (lactose intolerance, gastrointestinal infections, etc.) and patients with potential $\mathrm{CD}$ were also excluded. Eligible patients were then specifically asked whether in the previous year they suffered from gastrointestinal or non-gastrointestinal symptoms. More precisely, they were interviewed to verify whether they experienced not only the symptoms listed in the Celiac Symptoms Index but also the recurrence of those symptoms that the patient complained of at the time of the initial diagnosis of $\mathrm{CD}^{(20)}$.

Patients who reported the symptoms were enroled in the study as cases, regardless of the severity and the frequency of these symptoms. Patients who denied the occurrence of any symptoms were enroled as controls. Cases and controls were then administered a form to record involuntary ingestion of gluten or to detect behaviours at risk of hypothetical gluten

Table 1. Form used to quantify the exposure to gluten in cases and controls

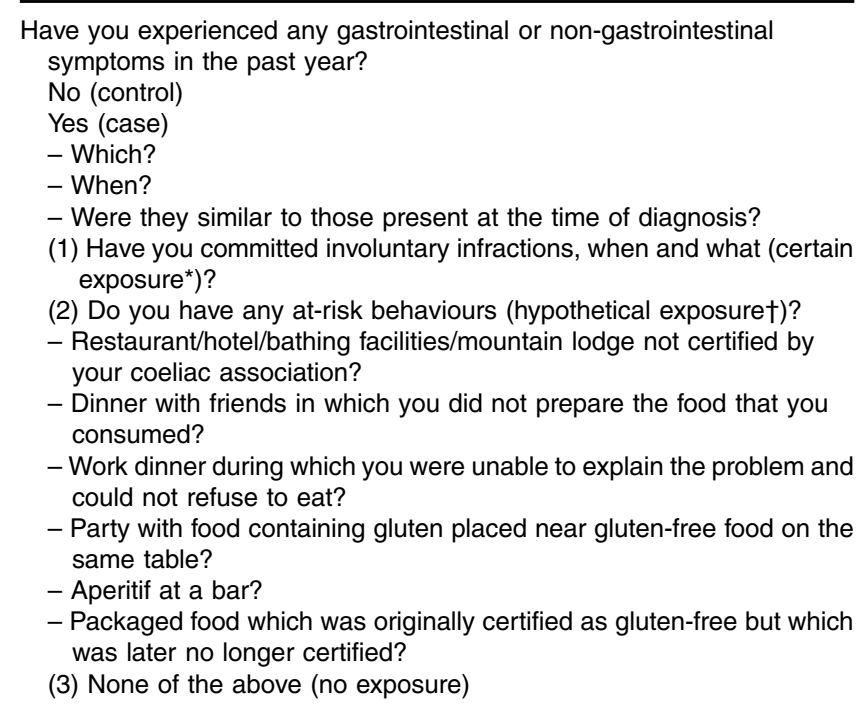

* Certain exposure, e.g. a patient orders a gluten-free pizza but after a taste realises that it contains gluten.

† Presumed exposure: the finding of even one of these situations is sufficient to consider the patient at risk of hypothetical exposure. 
exposure (Table 1). The form was specifically developed for this study and, although we developed it on the basis of our clinical experience and with the help of three coeliac patients who had been on a strict GFD for quite a few years, some of the points we took into account had already been considered by other authors ${ }^{(15,21)}$. The presence of any of the situations described in point 2 of Table 1 was sufficient to consider the patient at risk of hypothetical exposure to gluten; cases were asked whether the onset of symptoms was related to a gluten exposure, either certain or hypothetical. Sex, age at diagnosis of $\mathrm{CD}$, age at the time of our study, clinical presentation at time of diagnosis (classical, non-classical, asymptomatic $\mathrm{CD}^{(1)}$ ) and clinical picture at the time of re-evaluation are the demographic and clinical parameters collected for each patient.

\section{Statistics}

Continuous data with the mean and the standard deviation (age) or the median and 25th-75th percentiles were reported separately for cases and controls, which were compared with Student's $t$ test or the Mann-Whitney $U$ test, respectively. Categorical data as counts and percentage were compared with the Fisher exact test. A logistic model fit was used to assess the association of the type of gluten exposure and being a case, while adjusting for sex, age at diagnosis and duration of GDF (log transformed). Model statistics (goodnessof-fit test and model area under the receiver operating characteristic curve) were computed and also reported OR and 95\% CI. A subgroup analysis (using conditional logistic regression) was performed on those cases for whom an age- ( \pm 3 years) and sex-matched control is available. All analyses are done using Stata15 (StataCorp). A two-sided $P$ value $<0.05$ is considered statistically significant. In this proof of concept study, multiple tests corrections for post hoc comparisons were not applied.

\section{Sample size}

The sample size was determined by feasibility. Overall ninetyfive cases are available (no, hypothetical and certain exposure sample sizes were 25,57 and 13, respectively). The minimally detectable effect sizes for the three possible comparisons and for the comparison of aggregated groups are summarised in the online Supplementary Table. A two-sided $\alpha$ level of $5 \%$ and a power of $80 \%$ in the calculations were used. The power for detecting the observed effect sizes ( $\alpha 5 \%$ ) was computed.

\section{Ethics}

All patients signed informed consent before the biopsies for both clinical and research purposes. After verifying the quality, the data were all irreversibly anonymised. None of the patients had signed against participation in anonymous studies. The study was approved by the ethics committee of the Fondazione IRCCS Policlinico San Matteo according to the 1975 Declaration of Helsinki (6th revision, 2008).

\section{Results}

Between January 2017 and May 2018, a total of 125 adult patients with CD and on a GFD for at least a year attended our outpatient clinic. In all, ninety-five of them satisfied the enrolment criteria.

Of these ninety-five patients, fifty-two reported symptoms and they were enroled as cases; the remaining forty-three patients denied symptoms and were, therefore, enroled as controls (Table 2). Table 3 lists the symptoms found in the fiftytwo cases. In the majority of cases, these symptoms developed within 1 or $2 \mathrm{~d}$ after gluten exposure, either certain or hypothetical. According to the gluten-exposure form (Table 1), the ninety-five patients were divided into three different groups. In thirteen patients, an involuntary gluten exposure was certain; in fifty-seven patients no certain exposure was detected, but these patients had behaviours at risk of hypothetical gluten exposure; and in twenty-five patients there was no certain exposure nor behaviours at risk of hypothetical exposure.

Table 4 shows the prevalence of the three different groups of gluten exposure in cases and controls, together with the corresponding unadjusted and adjusted OR and 95\% CI. Although the comparison was not statistically significant, exposure was more frequent in controls ( $P=0.07$ in univariable analysis); post

Table 2. Demographic and clinical features of the fifty-two cases and forty-three controls (Numbers and percentages; mean values and standard deviations; medians and 25th-75th percentiles)

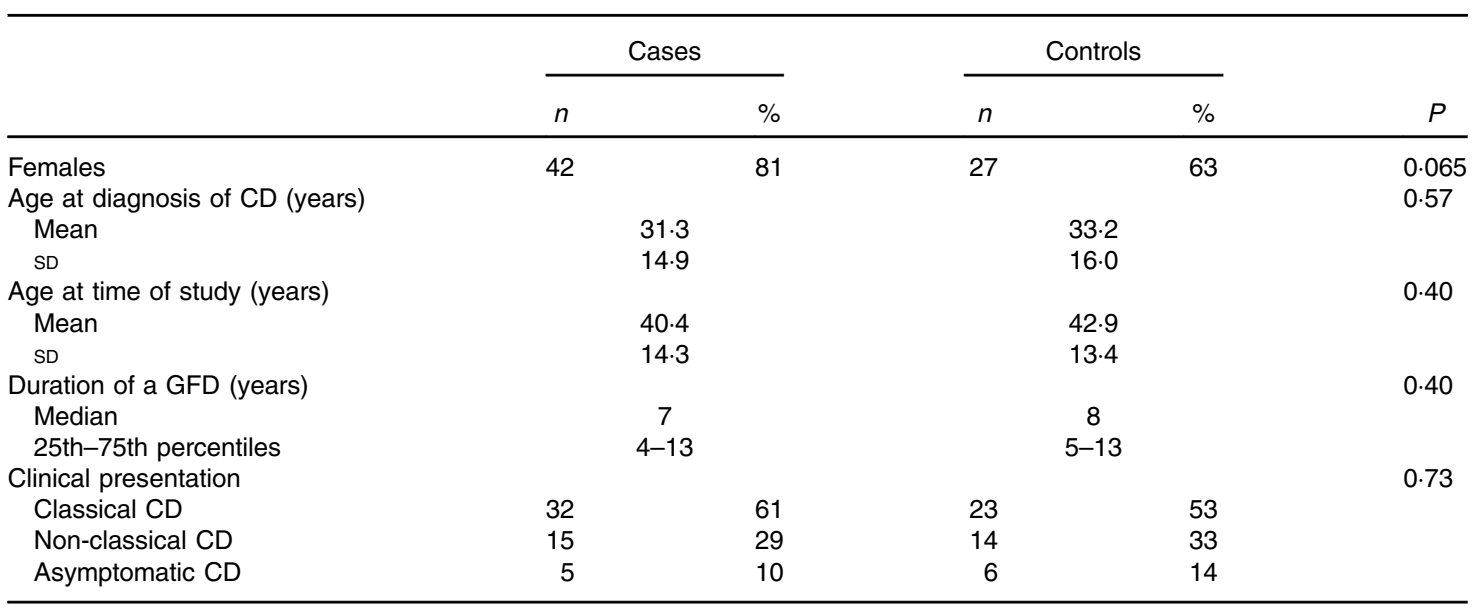

$C D$, coeliac disease; GFD, gluten-free diet. 
Table 3. Symptoms found during the clinical evaluation of patients affected by coeliac disease*

\begin{tabular}{lc}
\hline Symptoms & Number of episodes \\
\hline Abdominal pain & 27 \\
Diarrhoea & 24 \\
Vomiting & 8 \\
Nausea & 5 \\
Epigastric pain & 5 \\
Dermatitis herpetiformis & 3 \\
Oral aphthous ulcers & 3 \\
Headache & 1 \\
Sweating & 1 \\
Dyspepsia & 1 \\
\hline * Since each case could complain of more than one symptom, the total sum is greater \\
$\quad$ than the number of cases.
\end{tabular}

hoc comparisons show that this difference could be due to a higher prevalence of the 'no exposure' group in cases as contrasted to a higher prevalence of 'hypothetical exposure' in controls $(P=0 \cdot 031)$. A matched analysis for sex and age at diagnosis of CD did not show any difference at all. The multivariable logistic analysis confirms these findings, with OR of 0.38 and 0.58 for the 'hypothetical' and the 'certain exposure' as compared with the 'no exposure' category $(P=0 \cdot 134)$.

To increase the statistical power of our study, the 'certain exposure' group and the 'hypothetical exposure' one were grouped together and compared with the 'no exposure' group (Table 5). The exposure ('certain/hypothetical exposure') was more frequent in controls than in cases, with significance reached at the univariable logistic regression $(P=0 \cdot 048)$, but not with the Fisher's exact test $(P=0.06)$. The multivariable logistic analysis confirms these findings of borderline nonsignificance as well, with an OR of $0.38(P=0.068)$, for the 'hypothetical/certain exposure' as compared with the 'no exposure category'. This difference is also not statistically significant in a matched analysis.

We likewise grouped the 'no exposure' group with the 'hypothetical exposure' and compared them with the 'certain exposure' one. No difference in proportions was found (pre-

Table 4. Prevalence of cases and controls in the three groups of gluten exposure*

\begin{tabular}{|c|c|c|c|c|c|c|c|c|c|}
\hline & \multicolumn{2}{|c|}{ Controls } & \multicolumn{2}{|c|}{ Cases } & \multicolumn{2}{|c|}{ Unadjusted } & \multicolumn{2}{|c|}{ Adjusted } & \multirow[b]{2}{*}{ Adjusted $P$} \\
\hline & $n$ & $\%$ & $n$ & $\%$ & OR & $95 \% \mathrm{Cl}$ & OR & $95 \% \mathrm{Cl}$ & \\
\hline Type of exposure & & & & & & & & & 0.134 \\
\hline No exposure & 7 & 16 & 18 & 35 & 1 & & 1 & & \\
\hline Hypothetical exposure & 31 & 72 & 26 & 50 & 0.33 & $0.12,0.90$ & 0.34 & $0.11,0.99$ & 0.049 \\
\hline Certain exposure & 5 & 12 & 8 & 15 & 0.52 & $0.15,2.57$ & 0.58 & $0.13,2.57$ & 0.475 \\
\hline Male & 16 & 37 & 10 & 19 & 0.40 & $0.16,1.01$ & 0.43 & $0.16,1 \cdot 16$ & 0.096 \\
\hline Age at diagnosis (years) & & & & & 0.99 & $0.97,1.02$ & 0.98 & $0.95,1.01$ & 0.134 \\
\hline Mean & \multicolumn{2}{|c|}{33.2} & \multicolumn{2}{|c|}{$31 \cdot 3$} & & & & & \\
\hline SD & \multicolumn{2}{|c|}{$16 \cdot 0$} & \multicolumn{2}{|c|}{14.9} & & & & & \\
\hline Years of gluten-free dietł & & & & & 0.77 & $0.44,1.32$ & 0.59 & $0.31,1 \cdot 12$ & 0.109 \\
\hline Mean & \multicolumn{2}{|c|}{9.7} & \multicolumn{2}{|c|}{$8 \S$} & & & & & \\
\hline SD & \multicolumn{2}{|c|}{6.7} & \multicolumn{2}{|c|}{$5-11 \S$} & & & & & \\
\hline
\end{tabular}

* Univariable (unadjusted) and multivariable (adjusted) analysis. Multivariable model $P=0.049$, goodness of fit test $P=0.293$, area under receiver operating characteristic curve $=0.70$.

† The unadjusted $P$ value for type of exposure is 0.071

¥ Years of gluten-free diet was log-transformed before fitting the logistic model.

$\S$ Median and 25th-75th percentiles.

Table 5. Prevalence of cases and controls in the 'no exposure' group compared with the 'certain exposure' and 'hypothetical exposure' groups united together ${ }^{\star}$

\begin{tabular}{|c|c|c|c|c|c|c|c|c|c|}
\hline & \multicolumn{2}{|c|}{ Controls } & \multicolumn{2}{|c|}{ Cases } & \multicolumn{2}{|c|}{ Unadjusted } & \multicolumn{2}{|c|}{ Adjusted } & \multirow[b]{2}{*}{ Adjusted $P$} \\
\hline & $n$ & $\%$ & $n$ & $\%$ & OR & $95 \% \mathrm{Cl}$ & OR & $95 \% \mathrm{Cl}$ & \\
\hline Type of exposure $\dagger$ & & & & & & & & & 0.068 \\
\hline No exposure & 7 & 16 & 18 & 35 & 1 & & 1 & & \\
\hline Certain/hypothetical exposure & 36 & 84 & 34 & 65 & 0.37 & $0.14,0.99$ & 0.38 & $0.13,1.08$ & 0.068 \\
\hline Male & 16 & 37 & 10 & 19 & 0.40 & $0.16,1.01$ & 0.41 & $0.15,1.11$ & 0.079 \\
\hline Age at diagnosis (years) & & & & & 0.99 & $0.97,1.02$ & 0.98 & $0.95,1.01$ & 0.141 \\
\hline Mean & \multicolumn{2}{|c|}{$33 \cdot 2$} & \multicolumn{2}{|c|}{$31 \cdot 3$} & & & & & \\
\hline $\mathrm{SD}$ & \multicolumn{2}{|c|}{$16 \cdot 0$} & \multicolumn{2}{|c|}{14.9} & & & & & \\
\hline Years of gluten-free diet $\ddagger$ & \multirow{2}{*}{\multicolumn{2}{|c|}{9.7}} & & & 0.77 & $0.44,1.32$ & 0.58 & $0.31,1 \cdot 10$ & 0.097 \\
\hline Mean & & & \multicolumn{2}{|c|}{$8 \S$} & & & & & \\
\hline SD & \multicolumn{2}{|c|}{$6 \cdot 7$} & \multicolumn{2}{|c|}{$5-11 \S$} & & & & & \\
\hline
\end{tabular}

* Univariable (unadjusted) and multivariable (adjusted) analysis. Multivariable model $P=0.034$, goodness of fit test $P=0.288$, area under receiver operating characteristic curve $=0.70$

$\dagger$ The unadjusted $P$ value for type of exposure is 0.048 .

¥ Years of gluten-free diet was log-transformed before fitting the logistic model.

$\S$ Median and 25th-75th percentiles. 
valence of 'not exposed' $88 v .85 \%$ in cases and controls, respectively, $P=0.77)$. Finally, although females were more frequent among cases than controls $(42 / 52 v .27 / 43)$, this difference was not statistically significant (Table 2, $P=0 \cdot 06$ ). As far as age at diagnosis, clinical type of $\mathrm{CD}$, type of symptoms at the time of study and duration of GFD are concerned, no differences were found in univariable analysis. In multivariable analyses, none of the potential confounders as included in the logistic models, sex, age at diagnosis and duration of GDF, were significantly associated with the outcome (Tables 4 and 5).

Power analysis for the observed differences, given the available sample size is summarised in the online Supplementary Table. Power ranges between 5 and $53 \%$.

\section{Discussion}

To follow a strict and lifelong GFD is not an easy task. Wheat is certainly one of the cornerstones of both the Mediterranean and the Western diet, the two most common diets in Western countries. Moreover, gluten can be found in processed and packed food that should not contain gluten. The quantity of gluten found in these products can be so high that it could imply the ingestion of a significantly dangerous quantity of gluten ${ }^{(22)}$. The issue of possible contamination is therefore a very important problem which has been considered from not only a scientific point of view but also a legislative one by directives mandating the labelling of packaged foodstuffs ${ }^{(15,22,23)}$. Several studies investigated the capability of minimal quantities of gluten to induce intestinal lesions in CD patients; according to a meta-analysis of these studies, $10 \mathrm{mg}$ of gluten is unable to induce significant intestinal lesions ${ }^{(14)}$. We showed that such a large quantity of gluten can be found only in food that cannot be consumed by accident and involuntarily ${ }^{(10)}$. However, probably because of an objective difficulty in 'translating' these small quantities of gluten in everyday terms, coeliac patients are often afraid of inadvertently consuming gluten and believe that that must be the cause of their symptoms ${ }^{(15)}$.

Our results do not support the hypothesis that the ingestion of minimal quantities of gluten can cause symptoms in coeliac patients who have been on a GFD for at least a year. On the contrary, our results suggest that there is no relationship between exposure to minimal quantities of gluten and onset of symptoms. We hypothesised a causal and dose-related relationship between exposure and outcome, and thus we would expect a gradient between exposure to gluten and the number of cases. This was not the case. Indeed we found an inverse, though not significant, relationship between a 'certain or hypothetical exposure' and reporting of symptoms, with a $60 \%$ lower likelihood of being a case if exposed at the multivariable logistic model. These results therefore seem to suggest that symptoms are more frequent in patients who follow a GFD very well and have neither involuntary exposures nor behaviours at risk of hypothetical gluten exposure. So, these symptoms are likely independent of the ingestion of gluten, and it could be precisely their presence that leads the patient to follow a GFD even more strictly and to avoid situations that may place them at risk of hypothetical gluten exposure. However, this is a proof of concept study, with low power. These hypotheses warrant further confirmation in independent case series.
Having studied only coeliac patients who had been on a GFD for at least 1 year and in whom a frank histological improvement was proven is certainly one of the strengths of our study. This excludes the possibility that symptoms were due to gross gluten contamination and unsatisfactory clinical/histological response. On the contrary, the small sample size might be a limitation of our study, reducing the power to elicit the hypothesised association. However, it is difficult to assume that a larger sample size would be able to revert the observed reversal relationship between exposure and outcome.

Although studying the cause of the symptoms in our patients was not one of our study objectives, having found a greater prevalence of females in cases with respect to controls leads us to consider the possibility that the nocebo effect may have a role in the development of symptoms ${ }^{(24)}$. Moreover, although difficult to be precisely ascertained, there may also be the possibility that symptoms were due to some non-gluten components of gluten-free foods, such as FODMAP (Fermentable, Oligo-, Di-, Mono-saccharides And Polyols) ${ }^{(25)}$. Recently, an Italian study demonstrated the beneficial role of a low FODMAP diet in patients with CD on a GFD and persistent abdominal symptoms ${ }^{(26)}$.

Our results could have important practical implications. The involuntary, and undiscovered, ingestion of gluten cannot be considered responsible for the appearance of symptoms in CD patients on a GFD. If these symptoms are clinically relevant, it is therefore necessary to look for their cause as they could be caused either by a condition associated with $\mathrm{CD}$, and not yet discovered, or by one of the fearsome complications of $\mathrm{CD}^{(26-28)}$. We conclude that the involuntary ingestion of minimal quantities of gluten does not have a role in the origin of symptoms in patients with CD on a GFD.

\section{Acknowledgements}

A. S. is supported by an Investigational Fellowship by Collegio Ghislieri, Pavia, Italy (Assegno di ricerca annuale per giovani ricercatori). F. B. is the guarantor of this article and he is accepting full responsibility for the conduct of the study. He has had access to the data and has control of the decision to publish.

Specific author contributions are as follows: A. S., C. K. and F. B. planned and designed the study; M. P. and V. B. collected the clinical data; C. K. performed the statistical analysis; S. M., A. S. and F. B. wrote the manuscript. All authors approved the final version of the article, including the authorship list.

The authors have no conflicts of interest.

\section{Supplementary material}

For supplementary material/s referred to in this article, please visit https://doi.org/10.1017/S0007114518003616

\section{References}

1. Ludvigsson JF, Bai JC, Biagi F, et al. (2014) Diagnosis and management of adult coeliac disease - guidelines from the British Society of Gastroenterology. Gut 63, 1210-1228.

2. Silvester JA, Weiten D, Graff LA, et al. (2016) Living glutenfree: adherence, knowledge, lifestyle adaptations and 
feelings towards a gluten-free diet. I Hum Nutr Diet 29 374-382.

3. Abdulkarim AS, Burgart LJ, See J, et al. (2002) Etiology of nonresponsive celiac disease: results of a systematic approach. Am J Gastroenterol 97, 2016-2021.

4. Kurien M, Trott N \& Sanders DS (2016) Long-term care for patients with coeliac disease in the UK: a review of the literature and future directions. J Hum Nutr Diet 29, 617-623.

5. Pietzak MM (2005) Follow-up of patients with celiac disease: achieving compliance with treatment. Gastroenterology $\mathbf{1 2 8}$, Suppl. 1, S135-S141.

6. Rubio-Tapia A, Rahim MW, See JA, et al. (2010) Mucosal recovery and mortality in adults with celiac disease after treatment with a gluten-free diet. Am I Gastroenterol 105, 1412-1420.

7. Tortora R, Imperatore N, Capone P, et al. (2014) The presence of anti-endomysial antibodies and the level of anti-tissue transglutaminases can be used to diagnose adult coeliac disease without duodenal biopsy. Aliment Pharmacol Ther $\mathbf{4 0}$, 1223-1229.

8. Reilly NR, Husby S, Sanders DS, et al. (2018) Coeliac disease: to biopsy or not? Nat Rev Gastroenterol Hepatol 15, 60-66.

9. Troncone R, Mayer M, Spagnuolo F, et al. (1995) Endomysial antibodies as unreliable markers for slight dietary transgressions in adolescents with celiac disease. J Pediatr Gastroenterol Nutr 21, 69-72.

10. Biagi F, Andrealli A, Bianchi PI, et al. (2009) A gluten-free diet score to evaluate dietary compliance in patients with coeliac disease. BrJ Nutr 102, 882-887.

11. Biagi F, Bianchi PI, Marchese A, et al. (2012) A score that verifies adherence to a gluten-free diet: a cross-sectional, multicentre validation in real clinical life. Br J Nutr 108, 1884-1888.

12. Leffler DA, Dennis M, Edwards George JB, et al. (2009) A simple validated gluten-free diet adherence survey for adults with celiac disease. Clin Gastroenterol Hepatol 7, 530-536, 536.e1-e24.

13. Catassi C, Fabiani E, Iacono G, et al. (2007) A prospective, double-blind, placebo-controlled trial to establish a safe gluten threshold for patients with celiac disease. Am J Clin Nutr $\mathbf{8 5}$, 160-166.

14. Akobeng AK \& Thomas AG (2008) Systematic review: tolerable amount of gluten for people with coeliac disease. Aliment Pharmacol Ther 27, 1044-1052.

15. Silvester JA, Graff LA, Rigaux L, et al. (2016) Symptomatic suspected gluten exposure is common among patients with coeliac disease on a gluten-free diet. Aliment Pharmacol Ther 44, 612-619.

16. Kurada S, Yadav A \& Leffler DA (2016) Current and novel therapeutic strategies in celiac disease. Expert Rev Clin Pharmacol 9, 1211-1223.

17. Biagi F, Campanella J, Martucci S, et al. (2004) A milligram of gluten a day keeps the mucosal recovery away. Nutr Rev $\mathbf{6 2}$, 360-363.

18. Biagi F, Pezzimenti D, Campanella J, et al. (2001) Endomysial and tissue transglutaminase antibodies in coeliac sera. A comparison not influenced by previous serological testing. Scand J Gastroenterol 36, 955-958.

19. Biagi F, Vattiato C, Agazzi S, et al. (2014) A second duodenal biopsy is necessary in the follow-up of adult coeliac patients. Ann Med 46, 430-433.

20. Leffler DA, Dennis M, Edwards George J, et al. (2009) A validated disease-specific symptom index for adults with celiac disease. Clin Gastroenterol Hepatol 7, 1328-1334.

21. Leffler DA, Edwards-George J, Dennis M, et al. (2008) Factors that influence adherence to a gluten-free diet in adults with celiac disease. Dig Dis Sci 53, 1573-1581.

22. Ciacci C \& Mazzacca G (1998) Unintentional gluten ingestion in celiac patients. Gastroenterology 115, 243.

23. The European Parliament and the Council of the European Union; for the European Parliament, the president P. Cox; for the Council, the president A. Marzano. Directive 2003/89 EC of the European Parliament and of the Council of 10 November 2003 amending Directive 2000/13/EC as regards indication of the ingredients present in foodstuffs. Official J Eur Union L308, 15-18.

24. Vambheim SM \& Flaten MA (2017) A systematic review of sex differences in the placebo and the nocebo effect. $J$ Pain Res 10, 1831-1839.

25. Gibson PR, Muir JG \& Newnham ED (2015) Other dietary confounders: FODMAPS et al. Dig Dis 33, 269-276.

26. Testa A, Imperatore N, Rispo A, et al. (2018) Beyond irritable bowel syndrome: the efficacy of the low FODMAP diet for improving symptoms in inflammatory bowel diseases and celiac disease. Dig Dis 36, 271-280.

27. Fine KD, Meyer RL \& Lee EL (1997) The prevalence and causes of chronic diarrhea in patients with celiac sprue treated with a gluten-free diet. Gastroenterology 112, 1830-1838.

28. Leffler DA, Dennis M, Hyett B, et al. (2007) Etiologies and predictors of diagnosis in nonresponsive celiac disease. Clin Gastroenterol Hepatol 5, 445-450. 